\title{
On some explicit bounds of integral inequalities related to time scales
}

Zareen A. Khan ${ }^{1 *}$

\section{"Correspondence:}

dr.zareenkhan@ymail.com

'Department of Mathematics, Princess Nourah bint Abdul Rahman University, Riyadh, Kingdom of Saudi Arabia

\section{Springer}

\begin{abstract}
In this paper, we propose a new method to unify and extend some nonlinear dynamic double integral inequalities of two independent variables involving pairs of time scales via nabla derivative. The acquired results give explicit bounds which can be utilized to consider the subjective and quantitative properties of specific classes of dynamic conditions on time scales. An application to prove the validity of our established results is also given.
\end{abstract}

MSC: 26D15; 26D20; 39A12

Keywords: Gronwall's inequality; Time scales; Nabla differentiation; Dynamic equations

\section{Introduction}

Whenever there is a discussion about the importance of research work for boundedness, global existence, the stability of the solutions of differential and basic conditions of integral equations, the fact cannot be denied that Gronwall-Bellman inequality, Bihari inequality, and their various generalizations play a pivotal role in providing precise bounds of differential, integral, and also of difference equations. After the disclosure of the inequality coming about because of Gronwall, various mathematicians have showed significant enthusiasm to sum up the original form of this inequality, for which we may refer to $[5,12$, $16,17,25]$ and the references cited therein. On the other hand, Hilger [15], a nominee of the prime researcher, initiated the development of calculus of time scales to unify and extend different theories of both difference and differential equations in a consistent manner. Over the past few years, many authors came ahead and did a comprehensive analysis of the properties and utilization of different types of dynamic equations and inequalities on time scale [1, 2, 7-11, 14, 20, 22, 26-28]. At the beginning, Bohner and Peterson [6] examined the integral inequality on time scales of the form

$$
x(u) \leq a(u)+p(u) \int_{u_{0}}^{u} k(u, \tau)[b(\tau) x(\tau)+q(\tau)] \Delta \tau .
$$

After that, in 2009, Li [19] established the following integral inequality:

$$
x(u) \leq x_{0}+\int_{u_{0}}^{u}[f(\tau) x(\tau)+p(\tau)] \Delta \tau+\int_{u_{0}}^{u} f(\tau)\left[\int_{u_{0}}^{\tau} m(\tau, v) x(v) \Delta v\right] \Delta \tau
$$

(c) The Author(s) 2019. This article is distributed under the terms of the Creative Commons Attribution 4.0 International License (http://creativecommons.org/licenses/by/4.0/), which permits unrestricted use, distribution, and reproduction in any medium, provided you give appropriate credit to the original author(s) and the source, provide a link to the Creative Commons license, and indicate if changes were made. 
such that $m(\tau, v) \geq 0, m^{\Delta}(\tau, v) \geq 0$ for $u, v \in \mathbb{T}$ and $v \leq u$. Li [21] further studied the generalization of the nonlinear integral inequality of two independent variables on time scales as follows:

$$
x^{\alpha}(u, v) \leq a(u, v)+b(u, v) \int_{u_{0}}^{u} \int_{v_{0}}^{v}\left[g(\tau, \eta) x^{\alpha}(\tau, \eta)+h(\tau, \eta) x(\tau, \eta)\right] \Delta \eta \Delta \tau,
$$

with $\alpha>1$ being a real constant. Recently, Mi [23] proved Gronwall-Bellman delay integral inequality with two independent variables on time scales of the type

$$
\begin{aligned}
x^{\alpha}(u, v) \leq & a(u, v)+\int_{u_{0}}^{u} \int_{v_{0}}^{v} f_{1}(u, v, s, t) r_{1}\left(x\left(\phi_{1}(s), \tau_{1}(t)\right)\right) \Delta t \Delta s \\
& +\int_{u_{0}}^{u} \int_{v_{0}}^{v} f_{2}(u, v, s, t) r_{2}\left(x\left(\phi_{2}(s), \tau_{2}(t)\right)\right) \Delta t \Delta s,
\end{aligned}
$$

in which two nonlinear terms $r_{1}(x)$ and $r_{2}(x)$ are discussed, and these functions are not required to be nondecreasing. Based on the works of the above-mentioned researchers and using the same context of Gronwall-Bellman type inequalities, in this paper, we explore and generalize the following nonlinear dynamic inequalities of two independent variables via nabla derivative on time scales:

$$
\begin{aligned}
\Omega(x(u, v)) \leq & a(u, v)+\int_{u}^{\infty} \int_{v}^{\infty} \Omega^{\prime}(x(s, t)) f(s, t) \Psi(\Omega(x(s, t))) \nabla t \nabla s \\
& +L\left[\int_{u}^{\infty} g(s, v)\left(x(s, v)+\int_{s}^{\infty} \int_{v}^{\infty} h(\tau, \eta) \omega(x(\tau, \eta)) \nabla \eta \nabla \tau\right) \nabla s\right], \\
x(u, v) \leq & a(u, v) \\
& +\int_{u}^{\infty} \int_{v}^{\infty} f(s, t) L\left[x^{\alpha}(s, t)+\int_{s}^{\infty} \int_{t}^{\infty} g(\tau, \eta) L\left(x^{\beta}(\tau, \eta)\right) \nabla \eta \nabla \tau\right] \nabla t \nabla s \\
& +\int_{u}^{\infty} \int_{v}^{\infty} h(s, t) x(s, t) \nabla t \nabla s, \\
x^{\alpha}(u, v) \leq & a(u, v)+\int_{u}^{\infty} \int_{v}^{\infty} f(s, t) x^{\alpha}(s, t) \nabla t \nabla s \\
& +\int_{u}^{\infty} \int_{v}^{\infty} f(s, t)\left(\int_{s}^{\infty} \int_{t}^{\infty} g(\tau, \eta) x(\tau, \eta) \nabla \eta \nabla \tau\right) \\
& +L\left(\int_{u}^{\infty} h(s, v) \omega\left(x^{\alpha}(s, v)\right) \nabla s\right)
\end{aligned}
$$

for $(u, v) \in\left[u_{0}, \infty\right)_{\mathbb{T}} \times\left[u_{0}, \infty\right)_{\mathbb{T}}$. The established inequalities can be utilized as convenient apparatuses in the examination of the subjective hypothesis of solutions of dynamic conditions on time scales. It is assumed that the reader must be familiar with the knowledge and basic concepts of the calculus on time scales. For incredible research on the theory of time scales, we suggest the peruser to [6] and the paper presenting nabla subsidiaries by Atici and Guseinov [4].

\section{Nonlinear inequalities on time scales}

Throughout this work, denote $\mathbb{R}_{+}=[0, \infty)$ and $u_{0} \in \mathbb{T}$. $C_{\text {rd }}$ denotes the set of rdcontinuous functions. A time scale $\mathbb{T}$ is an arbitrary nonempty closed subset of the 
real numbers $\mathbb{R}$. $\Re$ denotes the set of all regressive and rd-continuous functions and $\Re^{+}=\{p \in \Re: 1+\mu(u) p(u)>0, u \in \mathbb{T}\}$.

Now, some essential definitions and lemmas on time scales, which will be used later in proofs of the present paper, are given below.

Definition 2.1 ([24]) If $\xi: \mathbb{R}_{+} \rightarrow \mathbb{R}_{+}$is a nondecreasing function and satisfies

(1) $\xi(z)$ is positive, nondecreasing, and continuous for $z>0$,

(2) $\frac{1}{r} \xi(z) \leq \xi\left(\frac{r}{z}\right)$ for $z \geq 0, r \geq 1$,

then it is said to belong to the class $J$.

Lemma 2.2 ([3]) If $a \in \mathfrak{R}, u_{0} \in \mathbb{T}$, then the exponential function $e_{a}\left(u, u_{0}\right)$ is the unique solution of the following initial value problem:

$$
\left\{\begin{array}{l}
x^{\Delta}(u)=a(u) x(u), \\
x\left(u_{0}\right)=1
\end{array}\right.
$$

To prove our main results, we first list the following assumptions:

(H1) $x(u, v), h(u, v), f(u, v), a(u, v), g(u, v)$ are nonnegative left dense continuous functions defined for $(u, v) \in\left[u_{0}, \infty\right)_{\mathbb{T}} \times\left[u_{0}, \infty\right)_{\mathbb{T}}$.

(H2) $a(u, v)$ is nonincreasing in both $u$ and $v$ and not identically zero.

(H3) $\omega(x)$ is a continuous, nondecreasing, subadditive, and submultiplicative function defined on $\mathbb{R}_{+}$with $\omega(x)>0$ on $[0, \infty)$.

(H4) $\Psi \in J$ and $\Omega \in C^{l}\left(\mathbb{R}_{+}, \mathbb{R}_{+}\right)$are subadditive and submultiplicative with $\Omega^{\prime}(x)>0$, $x>0$, (prime stands for the traditional derivative).

(H5) $L(x) \geq 0$ is a nondecreasing and continuous function on $\mathbb{R}_{+}$with $L(0)=0$.

Theorem 2.3 Assume that conditions (H1)-(H5) and relation (1) hold. Then

$$
\begin{aligned}
x(u, v) \leq & q(u, v) \Omega^{-1}\left[a(u, v)+L\left[F^{-1}[F(B(u, v))\right.\right. \\
& \left.\left.\left.+\int_{u}^{\infty} g(s, v)\left[q(s, v)+\int_{s}^{\infty} \int_{v}^{\infty} h(\tau, \eta) \omega(q(\tau, \eta)) \nabla \eta \nabla \tau\right] \nabla s\right]\right]\right]
\end{aligned}
$$

for $(u, v) \in\left[u_{0}, \infty\right)_{\mathbb{T}} \times\left[u_{0}, \infty\right)_{\mathbb{T}}$, where

$$
\begin{aligned}
& G(r)=\int_{r_{0}}^{r} \frac{\nabla s}{\Psi(\Omega(s))}, \quad r>0, r_{0}>0, \\
& F(z)=\int_{1}^{z} \frac{\nabla s}{\Omega^{-1}(L(s))+\omega\left(\Omega^{-1}(L(s))\right)}, \quad z>0 .
\end{aligned}
$$

The inverse functions of $F, G$ are $F^{-1}, G^{-1}$ respectively, and $u^{*}, v^{*} \in\left[u_{0}, \infty\right)_{\mathbb{T}}$ are chosen so that

$$
\begin{aligned}
& F(B(u, v))+\int_{u}^{\infty} g(s, v)\left[q(s, v)+\int_{s}^{\infty} \int_{v}^{\infty} h(\tau, \eta) \omega(q(\tau, \eta)) \nabla \eta \nabla \tau\right] \nabla s \in \operatorname{Dom}\left(F^{-1}\right), \\
& G\left(\Omega^{-1}(1)\right)+\int_{u}^{\infty} \int_{v}^{\infty} \Omega^{\prime}\left(\Omega^{-1}(p(s, t))\right) f(s, t) \nabla t \nabla s \in \operatorname{Dom}\left(G^{-1}\right),
\end{aligned}
$$




$$
\begin{aligned}
B(u, v)= & \int_{u}^{\infty} g(s, v)\left[q(s, v) \Omega^{-1}(a(s, v))\right. \\
& \left.+\int_{s}^{\infty} \int_{v}^{\infty} h(\tau, \eta) \omega(q(\tau, \eta)) \omega\left(\Omega^{-1}(a(\tau, \eta))\right) \nabla \eta \nabla \tau\right] \nabla s, \\
q(u, v)= & G^{-1}\left[G\left(\Omega^{-1}(1)\right)+\int_{u}^{\infty} \int_{v}^{\infty} \Omega^{\prime}\left(\Omega^{-1}(p(s, t))\right) f(s, t) \nabla t \nabla s\right] .
\end{aligned}
$$

Proof Denote a function $p(u, v)$ by

$$
p(u, v)=a(u, v)+L\left[\int_{u}^{\infty} g(s, v)\left[x(s, v)+\int_{s}^{\infty} \int_{v}^{\infty} h(\tau, \eta) \omega(x(\tau, \eta)) \nabla \eta \nabla \tau\right] \nabla s\right],
$$

then (1) gives

$$
\Omega(x(u, v)) \leq p(u, v)+\int_{u}^{\infty} \int_{v}^{\infty} \Omega^{\prime}(x(s, t)) f(s, t) \Psi(\Omega(x(s, t))) \nabla t \nabla s .
$$

Since $p(u, v) \geq 1$ is nonincreasing in $u$ and by Definition 2.1 for $\Psi,(10)$ takes the form

$$
\frac{\Omega(x(u, v))}{p(u, v)} \leq 1+\int_{u}^{\infty} \int_{v}^{\infty} \Omega^{\prime}(x(s, t)) f(s, t) \Psi\left(\frac{\Omega(x(s, t))}{p(s, t)}\right) \nabla t \nabla s .
$$

Denoting the right-hand side of $(11)$ by $m(u, v)$, we obtain

$$
m(u, v)=1+\int_{u}^{\infty} \int_{v}^{\infty} \Omega^{\prime}(x(s, t)) f(s, t) \Psi\left(\frac{\Omega(x(s, t))}{p(s, t)}\right) \nabla t \nabla s .
$$

Clearly $\Omega^{-1}$ is subadditive and submultiplicative, then from (11) and (12), we have

$$
\frac{\Omega(x(u, v))}{p(u, v)} \leq m(u, v)
$$

which gives

$$
x(u, v) \leq \Omega^{-1}(p(u, v)) \Omega^{-1}(m(u, v)) .
$$

Taking nabla derivative of $m(u, v)$ with respect to $u$ and by using (13) and (14), we deduce

$$
m^{\nabla u}(u, v) \geq-\int_{v}^{\infty} \Omega^{\prime}\left(\Omega^{-1}(p(u, t))\right) \Omega^{\prime}\left(\Omega^{-1}(m(u, t))\right) f(u, t) \Psi(m(u, t)) \nabla t
$$

Since $m(u, t) \leq \Omega^{-1}(m(u, t)) \leq \Omega^{-1}(m(u, v))$ for all $t \in[v, \infty)_{\mathbb{T}}$, the above inequality implies that

$$
\frac{m^{\nabla u}(u, v)}{\Omega^{\prime}\left(\Omega^{-1}(m(u, v))\right)} \geq-\int_{v}^{\infty} \Omega^{\prime}\left(\Omega^{-1}(p(u, t))\right) f(u, t) \Psi(m(u, t)) \nabla t .
$$

Now consider $\left[\Omega^{-1}(m(u, v))\right]^{\nabla u}$. By applying differential mean theorem on $\Omega$ for some $\sigma \in \mathbb{T}$ between $m(u, v)$ and $m(\mu(u), v)$, we have

$$
\left[\Omega^{-1}(m(u, v)]^{\nabla u}=\frac{m^{\nabla u}(u, v)}{\Omega^{\prime}\left(\Omega^{-1}(\sigma)\right)} \leq \frac{m^{\nabla u}(u, v)}{\Omega^{\prime}\left(\Omega^{-1}(m(u, v))\right)} .\right.
$$


From (15) and (16), it is easy to observe that

$$
\left[\Omega^{-1}(m(u, v))\right]^{\nabla u} \geq-\int_{v}^{\infty} \Omega^{\prime}\left(\Omega^{-1}(p(u, t))\right) f(u, t) \Psi(m(u, t)) \nabla t .
$$

Nabla integrating the above inequality from $u$ to $\infty$ by keeping $v$ fixed and using the fact that $m(\infty, v)=1$, we get

$$
\Omega^{-1}(m(u, v)) \leq y(u, v)
$$

where

$$
y(u, v)=\Omega^{-1}(1)+\int_{u}^{\infty} \int_{v}^{\infty} \Omega^{\prime}\left(\Omega^{-1}(p(s, t))\right) f(s, t) \Psi(m(s, t)) \nabla t \nabla s .
$$

By the definition of $y(u, v)$ and using (17), we have

$$
y^{\nabla u}(u, v) \geq-\int_{v}^{\infty} \Omega^{\prime}\left(\Omega^{-1}(p(u, t))\right) f(u, t) \Psi(\Omega(v(u, t))) \nabla t .
$$

Since $\Psi$ is nondecreasing and $y(u, t) \leq \Omega(y(u, t)) \leq \Omega(y(u, v))$ for all $t \in[v, \infty)_{\mathbb{T}}$, the above relation implies that

$$
\frac{y^{\nabla u}(u, v)}{\Psi(\Omega(y(u, v)))} \geq-\int_{v}^{\infty} \Omega^{\prime}\left(\Omega^{-1}(p(u, t))\right) f(u, t) \nabla t .
$$

Now consider

$$
\begin{aligned}
G^{\nabla u}(y(u, v)) & =\left(\int_{y_{0}}^{y(u, v)} \frac{\nabla r}{\Psi(\Omega(r))}\right)^{\nabla u}=\lim _{\sigma \rightarrow u} \frac{1}{\mu(u)-\sigma} \int_{y(\sigma, v)}^{y(\mu(u), v)} \frac{\nabla r}{\Psi(\Omega(r))} \\
& \leq \lim _{\sigma \rightarrow u} \frac{1}{\mu(u)-\sigma} \int_{y(\sigma, v)}^{y(\mu(u), v)} \nabla r \cdot \frac{1}{\Psi(\Omega(r))}=\frac{y^{\nabla u}(u, v)}{\Psi(\Omega(y(u, v)))},
\end{aligned}
$$

as $\Psi$ is nondecreasing and $\Omega(y(u, v))>0$. By (18)

$$
G^{\nabla u}(y(u, v)) \geq-\int_{v}^{\infty} \Omega^{\prime}\left(\Omega^{-1}(p(u, t))\right) f(u, t) \nabla t .
$$

Keeping $v$ fixed, nabla integrating the above inequality from $u$ to $\infty$, and using the fact that $y(\infty, v)=\Omega^{-1}(1)$, we get

$$
y(u, v)=q(u, v)
$$

such that $q(u, v)$ is as given in (8). From (14), (17), and (20), we have

$$
x(u, v) \leq q(u, v) \Omega^{-1}(p(u, v)) .
$$

From (9) and (21), we deduce

$$
\begin{aligned}
& p(u, v)-a(u, v) \\
& \quad=L\left[\int_{u}^{\infty} g(s, v)\left[x(s, v)+\int_{s}^{\infty} \int_{v}^{\infty} h(\tau, \eta) \omega(x(\tau, \eta)) \nabla \eta \nabla \tau\right] \nabla s\right]
\end{aligned}
$$




$$
\begin{aligned}
\leq & L\left[\int _ { u } ^ { \infty } g ( s , v ) \left[q(s, v) \Omega^{-1}(p(s, v))+\int_{s}^{\infty} \int_{v}^{\infty} h(\tau, \eta) \omega(q(\tau, \eta)\right.\right. \\
& \left.\left.\left.\times \Omega^{-1}(p(\tau, \eta))\right) \nabla \eta \nabla \tau\right] \nabla s\right] \\
= & L\left[\int _ { u } ^ { \infty } g ( s , v ) \left[q(s, v) \Omega^{-1}(p(s, v))-q(s, v) \Omega^{-1}(a(s, v))+q(s, v)\right.\right. \\
& \times \Omega^{-1}(a(s, v))+\int_{s}^{\infty} \int_{v}^{\infty} h(\tau, \eta) \omega\left[q(\tau, \eta) \Omega^{-1}(p(\tau, \eta))\right. \\
& \left.\left.\left.-q(\tau, \eta) \Omega^{-1}(a(\tau, \eta))+q(\tau, \eta) \Omega^{-1}(a(\tau, \eta))\right] \nabla \eta \nabla \tau\right] \nabla s\right] \\
= & L\left[\int _ { u } ^ { \infty } g ( s , v ) \left[q(s, v) \Omega^{-1}(a(s, v))+\int_{s}^{\infty} \int_{v}^{\infty} h(\tau, \eta) \omega(q(\tau, \eta))\right.\right. \\
& \left.\times \omega\left(\Omega^{-1}(a(\tau, \eta))\right) \nabla \eta \nabla \tau\right] \nabla s+\int_{u}^{\infty} g(s, v)\left[q(s, v) \Omega^{-1}(p(s, v)-a(s, v))\right. \\
& \left.\left.+\int_{s}^{\infty} \int_{v}^{\infty} h(\tau, \eta) \omega(q(\tau, \eta)) \omega\left(\Omega^{-1}(p(\tau, \eta)-a(\tau, \eta))\right) \nabla \eta \nabla \tau\right] \nabla s\right] \\
\leq & L(r(x, y)),
\end{aligned}
$$

where

$$
\begin{aligned}
r(u, v)= & B(u, v)+\int_{u}^{\infty} g(s, v)\left[q(s, v) \Omega^{-1}(p(s, v)-a(s, v))\right. \\
& \left.+\int_{s}^{\infty} \int_{v}^{\infty} h(\tau, \eta) \omega(q(\tau, \eta)) \omega\left(\Omega^{-1}(p(\tau, \eta)-a(\tau, \eta))\right) \nabla \eta \nabla \tau\right] \nabla s,
\end{aligned}
$$

and $B(u, v)$ is as defined in (7). Fixing any two time-scale points $u_{1} \in\left[u^{\star}, \infty\right)_{\mathbb{T}}$ and $v_{1} \in$ $\left[v^{\star}, \infty\right)_{\mathbb{T}}$, then from $(22)$ and $(23)$ it is easy to observe that

$$
\begin{aligned}
r(u, v) \leq & B\left(u_{1}, v_{1}\right)+\int_{u}^{\infty} g(s, v)\left[q(s, v) \Omega^{-1}(L(r(s, v)))\right. \\
& \left.+\int_{s}^{\infty} \int_{v}^{\infty} h(\tau, \eta) \omega(q(\tau, \eta)) \omega\left(\Omega^{-1}(L(r(\tau, \eta)))\right) \nabla \eta \nabla \tau\right] \nabla s
\end{aligned}
$$

for $(u, v) \in\left[u_{1}, \infty\right)_{\mathbb{T}} \times\left[v_{1}, \infty\right)_{\mathbb{T}}$. Define $z(u, v)$ to the right-hand side of the last relation, therefore

$$
r(u, v) \leq z(u, v)
$$

Since $\omega$ is nondecreasing and $r(u, \eta) \leq z(u, \eta) \leq z(u, v)$ for all $\eta \in[v, \infty)_{\mathbb{T}}$, therefore, by the differentiation of $z(u, v)$ with respect to $u$, we have

$$
\frac{z^{\nabla u}(u, v)}{\Omega^{-1}(L(z))+\omega\left(\Omega^{-1}(L(z))\right)} \geq-g(u, v)\left[q(u, v)+\int_{u}^{\infty} \int_{v}^{\infty} h(s, \eta) \omega(q(s, \eta)) \nabla \eta \nabla s\right] .
$$

Consider $F^{\nabla u}(z(u, v))$ to be the same as in the derivation of (19) and from (25), we get

$$
F^{\nabla u}(z(u, v)) \geq-g(u, v)\left[q(u, v)+\int_{u}^{\infty} \int_{v}^{\infty} h(s, \eta) \omega(q(s, \eta)) \nabla \eta \nabla s\right]
$$


for fixed $v$. Nabla integrating the above inequality from $u$ to $\infty$ and using the fact that $z(\infty, v)=B\left(u_{1}, v_{1}\right)$, we have

$$
F(z(u, v)) \leq F\left(B\left(u_{1}, v_{1}\right)\right)+\int_{u}^{\infty} g(s, v)\left[q(s, v)+\int_{s}^{\infty} \int_{v}^{\infty} h(\tau, \eta) \omega(q(\tau, \eta)) \nabla \eta \nabla \tau\right] \nabla s .
$$

We observe that $r\left(u_{1}, v_{1}\right) \leq z\left(u_{1}, v_{1}\right)$ by setting $u=u_{1}, v=v_{1}$ in the last inequality, and

$$
\begin{aligned}
z\left(u_{1}, v_{1}\right) \leq & F^{-1}\left[F\left(B\left(u_{1}, v_{1}\right)\right)\right. \\
& \left.+\int_{u_{1}}^{\infty} g(s, v)\left[q(s, v)+\int_{s}^{\infty} \int_{v_{1}}^{\infty} h(\tau, \eta) \omega(q(\tau, \eta)) \nabla \eta \nabla \tau\right] \nabla s\right] .
\end{aligned}
$$

Also from (22)

$$
\begin{aligned}
p\left(u_{1}, v_{1}\right) \leq & a\left(u_{1}, v_{1}\right)+L\left[F ^ { - 1 } \left[F\left(B\left(u_{1}, v_{1}\right)\right)\right.\right. \\
& \left.\left.+\int_{u_{1}}^{\infty} g(s, v)\left[q(s, v)+\int_{s}^{\infty} \int_{v_{1}}^{\infty} h(\tau, \eta) \omega(q(\tau, \eta)) \nabla \eta \nabla \tau\right] \nabla s\right]\right] .
\end{aligned}
$$

The required estimate in (4) can be obtained from the above inequality and by the arbitrary nature of $u_{1}, v_{1}$ for $(u, v) \in\left[u_{1}, \infty\right)_{\mathbb{T}} \times\left[v_{1}, \infty\right)_{\mathbb{T}}$ in $(21)$.

Remark 2.4 As a special case of delta derivative on time scales, if $u$ is fixed, $\Omega(u)=\omega(u)=$ $u, a=u_{0}, f=0, L(u)=u, g=f, h=w(\tau, s)$ in Theorem 2.3, then it reduces to Theorem 3.1 due to Li [19] with $p(\tau)=0$.

In order to prove our next theorem, we establish the following interesting lemma.

Lemma 2.5 Let $l \geq 0, \alpha \geq \beta>0$, and $\alpha \neq 0$, then

$$
l^{\frac{\beta}{\alpha}} \leq \frac{\beta}{\alpha} l+\frac{\alpha-\beta}{\alpha} .
$$

Proof: Obviously the above inequality is valid if $\beta=0$. However, if $\beta>0$, let $\delta=\frac{\beta}{\alpha}$, then $\delta=1$ by ([13], Lemma 2.1), we have

$$
l^{\frac{\beta}{\alpha}} \leq \frac{\beta}{\alpha} M^{\frac{\beta-\alpha}{\alpha}} l+\frac{\alpha-\beta}{\alpha} M^{\frac{\beta}{\alpha}}
$$

for any $M>0$. Let $M=1$, we get (26).

Theorem 2.6 Assume that conditions (H1), (H2), (H5), and relation (2) hold with $\alpha \geq \beta>$ 0 and $L \in J$. Then

$$
\begin{aligned}
x(u, v) \leq & a(u, v)+Q(u, v)\left[1+\int_{u}^{\infty} \int_{v}^{\infty} f(s, t) d(s, t) e_{-S}^{\wedge}(t, \rho(s)) \nabla t \nabla s\right] \\
& \times\left[1+\alpha \int_{u}^{\infty} \int_{v}^{\infty} f(s, t) L\left(F _ { 1 } ^ { - 1 } \left(F_{1}(1)\right.\right.\right. \\
& \left.\left.\left.+\int_{s}^{\infty} \int_{t}^{\infty}\left(\alpha f(\tau, \eta)+\frac{\beta}{\alpha} g(\tau, \eta)\right) \nabla \eta \nabla \tau\right)\right) \nabla t \nabla s\right]
\end{aligned}
$$


for $(u, v) \in\left[u_{0}, \infty\right)_{\mathbb{T}} \times\left[u_{0}, \infty\right)_{\mathbb{T}}$, provided that $S=\int_{v}^{\infty} f(u, t) d(u, t) \nabla t$,

$$
\begin{aligned}
& d(u, v)= 1+\alpha \int_{u}^{\infty} \int_{v}^{\infty} f(s, t) L\left(F _ { 1 } ^ { - 1 } \left(F_{1}(1)\right.\right. \\
&\left.\left.+\int_{s}^{\infty} \int_{t}^{\infty}\left(\alpha f(\tau, \eta)+\frac{\beta}{\alpha} g(\tau, \eta)\right) \nabla \eta \nabla \tau\right)\right) \nabla t \nabla s, \\
& F_{1}(r)=\int_{r_{0}}^{r} \frac{\nabla s}{L(s)}, \quad r>0 .
\end{aligned}
$$

$F_{1}^{-1}$ is the inverse function of $F_{1}$, and $u^{*}, v^{*} \in\left[u_{0}, \infty\right)_{T}$ is chosen so that

$$
\begin{aligned}
& F_{1}(1)+\int_{u}^{\infty} \int_{v}^{\infty}\left(\alpha f(s, t)+\frac{\beta}{\alpha} g(s, t)\right) \nabla t \nabla s \in \operatorname{Dom}\left(F_{1}^{-1}\right), \\
& Q(u, v)= \int_{u}^{\infty} \int_{v}^{\infty} f(s, t) L[(1-\alpha)+\alpha(a(s, t)) \\
&\left.+\int_{s}^{\infty} \int_{t}^{\infty} g(\tau, \eta) L((1-\beta)+\beta(a(\tau, \eta))) \nabla \eta \nabla \tau\right] \nabla t \nabla s \\
&+\int_{u}^{\infty} \int_{v}^{\infty} h(s, t) a(s, t) \nabla t \nabla s,
\end{aligned}
$$

where $e^{\wedge}$ denotes the nabla exponential function.

Proof Let us define $z(u, v)$ by

$$
\begin{aligned}
z(u, v)= & \int_{u}^{\infty} \int_{v}^{\infty} f(s, t) L\left[x^{\alpha}(s, t)+\int_{s}^{\infty} \int_{t}^{\infty} g(\tau, \eta) L\left(x^{\beta}(\tau, \eta)\right) \nabla \eta \nabla \tau\right] \nabla t \nabla s \\
& +\int_{u}^{\infty} \int_{v}^{\infty} h(s, t) u(s, t) \nabla t \nabla s,
\end{aligned}
$$

then (2) can be restated as

$$
x(u, v) \leq a(u, v)+z(u, v) .
$$

Lemma 2.5 and (32) imply that

$$
x^{\alpha}(u, v) \leq \alpha(a(u, v)+z(u, v))+(1-\alpha)
$$

and

$$
x^{\beta}(u, v) \leq \beta(a(u, v)+z(u, v))+(1-\beta) .
$$

It is clear from (32)-(34) in (31) that

$$
\begin{aligned}
z(u, v) \leq & m(u, v) \\
& +\alpha \int_{u}^{\infty} \int_{v}^{\infty} f(s, t) L\left[z(s, t)+\frac{\beta}{\alpha} \int_{s}^{\infty} \int_{t}^{\infty} g(\tau, \eta) L(z(\tau, \eta)) \nabla \eta \nabla \tau\right] \nabla t \nabla s,
\end{aligned}
$$


provided that

$$
m(u, v)=Q(u, v)+\int_{u}^{\infty} \int_{v}^{\infty} h(s, t) z(s, t) \nabla t \nabla s
$$

and $Q(u, v)$ is as defined in (30). Obviously $m(u, v) \geq 1$ in nonincreasing in $u$ and from (35), we get

$$
z(u, v) \leq m(u, v) y(u, v)
$$

where

$$
y(u, v)=1+\alpha \int_{u}^{\infty} \int_{v}^{\infty} f(s, t) L\left[\frac{z(s, t)}{m(s, t)}+\frac{\beta}{\alpha} \int_{s}^{\infty} \int_{t}^{\infty} g(\tau, \eta) \frac{L(z(\tau, \eta))}{m(\tau, \eta)} \nabla \eta \nabla \tau\right] \nabla t \nabla s .
$$

Taking nabla derivative with respect to $u$, using (37) and Definition 2.1 in the above inequality, we deduce

$$
\begin{aligned}
y^{\nabla u}(u, v) & \geq-\alpha \int_{v}^{\infty} f(u, t) L\left[y(u, t)+\frac{\beta}{\alpha} \int_{u}^{\infty} \int_{t}^{\infty} g(s, \eta) L(y(s, \eta)) \nabla \eta \nabla s\right] \\
& \geq-\alpha \int_{v}^{\infty} f(u, t) L(r(u, t)) \nabla t
\end{aligned}
$$

such that $r(u, v)=y(u, v)+\frac{\beta}{\alpha} \int_{u}^{\infty} \int_{v}^{\infty} g(s, t) L(y(s, t)) \nabla t \nabla s$. By the definition of $r(u, v)$ and using (38), we have

$$
\frac{r^{\nabla u}(u, v)}{L(r(u, v))} \geq-\int_{v}^{\infty}\left(\alpha f(u, t)+\frac{\beta}{\alpha} g(u, t)\right) \nabla t
$$

for fixed $v$. Nabla integrating the last inequality from $u$ to $\infty$, using the fact that $r(\infty, v)=1$ and from (29), we obtain

$$
r(u, v) \leq F_{1}^{-1}\left[F(1)+\int_{u}^{\infty} \int_{v}^{\infty}\left(\alpha f(s, t)+\frac{\beta}{\alpha} g(s, t)\right) \nabla t \nabla s\right] .
$$

By substituting the above inequality in (38), nabla integrating again the resulting inequality from $u$ to $\infty$, using the fact that $y(\infty, v)=1$ and from (37), we get

$$
z(u, v) \leq m(u, v) d(u, v)
$$

where $d(u, v)$ is as mentioned in (28). Now consider (36) and let $b(u, v)=\int_{u}^{\infty} \int_{v}^{\infty} h(s, t) \times$ $z(s, t) \nabla t \nabla s$. Taking nabla derivative of $b(u, v)$ and (39), we have

$$
\begin{aligned}
b^{\nabla u}(u, v) & \geq-\int_{v}^{\infty} h(u, t) m(u, t) d(u, t) \\
& \geq-\int_{v}^{\infty} h(u, t) Q(u, t) d(u, t)-\int_{v}^{\infty} h(u, t) d(u, t) b(u, t) .
\end{aligned}
$$


Let $S=\int_{v}^{\infty} h(u, t) d(u, t) \nabla t$. Since it is assumed that $S>0$, then $-S \in \Re_{v}^{+}$and $e_{-S}^{\wedge}(u, \zeta)>0$ for all $u \in \mathbb{T}$ by Theorem 5.2 (vi) and (vii) [3]. Inequality (40) by utilizing the nabla quotient rule from Theorem 2.1 [18] and Lemma 2.2 implies that

$$
\left[b / e_{-S}^{\wedge}(u, \zeta)\right]^{\nabla u}=\int_{v}^{\infty}\left[b^{\nabla u}(u, t)+h(u, t) d(u, t) b(u, t)\right] / e_{-S}^{\wedge}(\rho(u), \zeta) \nabla t .
$$

Substituting $u$ with $s$, integrating the above equation from $\zeta$ to $\infty$, we get

$$
b(\zeta, v) e_{-S}^{\wedge}(\rho(u), \zeta) \leq \int_{\zeta}^{\infty} \int_{v}^{\infty} h(s, t) d(s, t) Q(s, t) / e_{-S}^{\wedge}(\rho(s), \zeta) \nabla t \nabla s .
$$

Since $\zeta$ is arbitrary, then substituting $\zeta$ with $u$ in (41), using (36), (40), and from [6] (Theorem 3.15 (iv) and (v)), we deduce

$$
\int_{u}^{\infty} \int_{v}^{\infty} h(s, t) d(s, t) b(s, t) \nabla t \nabla s \leq \int_{u}^{\infty} \int_{v}^{\infty} h(s, t) d(s, t) Q(s, t) e_{-S}^{\wedge}(\rho(s), \zeta) \nabla t \nabla s,
$$

which implies the estimation for $m(u, v)$ such that

$$
m(u, v) \leq Q(u, v)\left[1+\int_{u}^{\infty} \int_{v}^{\infty} h(s, t) d(s, t) e_{-S}^{\wedge}(\rho(s), \zeta) \nabla t \nabla s\right] .
$$

A combination of (32), (39), and (42) yields the desired bound in (27). The subdomain $u^{*}, v^{*} \in\left[u_{0}, \infty\right)_{T}$ is obvious.

Remark 2.7 As a special case of delta derivative on time scales, if we take $f=0, h=b$, and $a(u, v)$ is a nondecreasing function, then Theorem 2.6 changes to Theorem 1 due to $\mathrm{Li}$ [20].

Theorem 2.8 Assume that conditions (H1)-(H3), (H5) and relation (3) hold with $\alpha>1$. Then

$$
\begin{aligned}
x(u, v) \leq & q^{\frac{1}{\alpha}}(u, v) \\
& \times\left[a(u, v)+L\left(G ^ { - 1 } \left(G\left(\int_{u}^{\infty} h(s, v) \omega(a(s, v) q(s, v)) \nabla s\right)\right.\right.\right. \\
& \left.\left.\left.+\int_{u}^{\infty} h(s, v) \omega(q(s, v)) \nabla s\right)\right)\right]^{\frac{1}{\alpha}}
\end{aligned}
$$

for $(u, v) \in\left[u_{0}, \infty\right)_{\mathbb{T}} \times\left[u_{0}, \infty\right)_{\mathbb{T}}$, provided that $S=\frac{\alpha-1}{\alpha} \int_{v}^{\infty} f(u, t) \nabla t$,

$$
G(r)=\int_{r_{0}}^{r} \frac{\nabla s}{\omega(L(s))}, \quad r>0 .
$$

$G^{-1}$ is the inverse function of $G$, and $u^{*}, v^{*} \in\left[u_{0}, \infty\right)_{T}$ is chosen so that

$$
G\left(\int_{u}^{\infty} h(s, v) \omega(a(s, v) q(s, v)) \nabla s\right)+\int_{v}^{\infty} h(s, v) \omega(q(s, v)) \nabla s \in \operatorname{Dom}(G)^{-1},
$$




$$
\begin{aligned}
q(u, v)= & +\int_{u}^{\infty} \int_{v}^{\infty} f(s, t) \\
& \times\left(1+\frac{\alpha-1}{\alpha} \int_{s}^{\infty} \int_{t}^{\infty} g(\tau, \eta) n^{\frac{1-\alpha}{\alpha}}(\tau, \eta) e_{-S}^{\wedge}(\eta, \rho(\tau)) \nabla \eta \nabla \tau\right)^{\frac{\alpha}{\alpha-1}} \nabla t \nabla s,
\end{aligned}
$$

where $e^{\wedge}$ denotes the nabla exponential function.

Proof Denote (3) by a function of $z(u, v)$. Then

$$
x(u, v) \leq z^{\frac{1}{\alpha}}(u, v)
$$

furthermore,

$$
\begin{aligned}
z(u, v)= & n(u, v)+\int_{u}^{\infty} \int_{v}^{\infty} f(s, t) x^{\alpha}(s, t) \nabla t \nabla s \\
& +\int_{u}^{\infty} \int_{v}^{\infty} f(s, t)\left(\int_{s}^{\infty} \int_{t}^{\infty} g(\tau, \eta) x(\tau, \eta) \nabla \eta \nabla \tau\right) \nabla t \nabla s, \\
n(u, v)= & a(u, v)+L\left(\int_{u}^{\infty} h(s, v) \omega\left(x^{\alpha}(s, v)\right) \nabla s\right) .
\end{aligned}
$$

Since $n(u, v) \geq 1$ is nonincreasing in $u$ and from (46), (47), we get

$$
z(u, v) \leq n(u, v) y(u, v)
$$

where

$$
\begin{aligned}
y(u, v)= & +\int_{u}^{\infty} \int_{v}^{\infty} f(s, t) \frac{z(s, t)}{n(s, t)} \nabla t \nabla s \\
& +\int_{u}^{\infty} \int_{v}^{\infty} f(s, t)\left(\int_{s}^{\infty} \int_{t}^{\infty} g(\tau, \eta) n^{\frac{1-\alpha}{\alpha}}(\tau, \eta)\left(\frac{z(\tau, \eta)}{n(\tau, \eta)}\right)^{\frac{1}{\alpha}} \nabla \eta \nabla \tau\right) \nabla t \nabla s .
\end{aligned}
$$

Nabla deriving the above equation with respect to $u$ and using (49), we have

$$
\begin{aligned}
y^{\nabla u}(u, v) & \geq-\int_{v}^{\infty} f(u, t)\left(y(u, t)+\int_{u}^{\infty} \int_{t}^{\infty} g(s, \eta) n^{\frac{1-\alpha}{\alpha}}(s, \eta) y^{\frac{1}{\alpha}}(s, \eta) \nabla \eta \nabla s\right) \nabla t \\
& \geq-\int_{v}^{\infty} f(u, t) r(u, t) \nabla t
\end{aligned}
$$

such that

$$
r(u, v)=y(u, v)+\int_{u}^{\infty} \int_{v}^{\infty} g(s, t) n^{\frac{1-\alpha}{\alpha}}(s, t) y^{\frac{1}{\alpha}}(s, t) \nabla t \nabla s .
$$

Since $y(u, v) \leq r(u, v)$, it follows from the definition of $r(u, v)$ and (50) that

$$
r^{\nabla u}(u, v) \geq-\int_{v}^{\infty} f(u, t) r(u, t) \nabla t-\int_{v}^{\infty} g(u, t) n^{\frac{1-\alpha}{\alpha}}(u, t) r^{\frac{1}{\alpha}}(u, t) \nabla t .
$$


From (52), we consider the initial value problem of differential equation

$$
\left\{\begin{array}{l}
r_{1}^{\nabla u}(u, v)=\left(-\int_{v}^{\infty} f(u, t) r(u, t) \nabla t-\int_{v}^{\infty} g(u, t) n^{\frac{1-\alpha}{\alpha}}(u, t) \nabla t\right)\left(\frac{\alpha-1}{\alpha}\right) \\
r_{1}(\infty, v)=1
\end{array}\right.
$$

which, by following the same steps from (40)-(42) with suitable changes, yields the solution

$$
r_{1}(u, v) \leq 1+\frac{\alpha-1}{\alpha} \int_{u}^{\infty} \int_{v}^{\infty} g(s, t) n^{\frac{\alpha-1}{\alpha}}(s, t) e_{-S}^{\wedge}(t, \rho(s)) \nabla t \nabla s .
$$

Since $r_{1}(u, v) \leq r(u, v)$, then (53) and (54) give

$$
r(u, v) \leq\left(1+\frac{\alpha-1}{\alpha} \int_{u}^{\infty} \int_{v}^{\infty} g(s, t) n^{\frac{\alpha-1}{\alpha}}(s, t) e_{-S}^{\wedge}(t, \rho(s)) \nabla t \nabla s\right)^{\frac{\alpha}{\alpha-1}}
$$

Substituting the above inequality in (50), nabla integrating the resulting inequality from $u$ to $\infty$, using the fact that $y(\infty, v)=1$ and from (46), (49), we deduce

$$
x^{\alpha}(u, v) \leq n(u, v) q(u, v),
$$

where $q(u, v)$ is as defined in (45). Denote $b(u, v)=\int_{u}^{\infty} h(s, v) \omega\left(x^{\alpha}(s, v)\right) \nabla s$, then (48) takes the form

$$
n(u, v)=a(u, v)+L(b(u, v)) .
$$

Therefore, by using (56), we observe that

$$
\begin{aligned}
b(u, v) & \leq \int_{u}^{\infty} h(s, v) \omega(n(s, v) q(s, v)) \nabla s \\
& \leq \int_{u}^{\infty} h(s, v) \omega(n(s, v)) \omega(q(s, v)) \nabla s \\
& \leq \int_{u}^{\infty} h(s, v) \omega(q(s, v)(a(s, v)+L(b(s, v)))) \nabla s \\
& \leq \int_{u}^{\infty} h(s, v) \omega(a(s, v) q(s, v)) \nabla s+\int_{u}^{\infty} h(s, v) \omega(q(s, v)) \omega(L(b(s, v))) \nabla s .
\end{aligned}
$$

Clearly, $\omega$ is a subadditive and submultiplicative function. Define $m(u, v)$ to be the righthand side of the last inequality, then $m(\infty, v)=\int_{u}^{\infty} h(s, v) \omega(a(s, v) q(s, v)) \nabla s, b(u, v) \leq$ $m(u, v)$, and by the definition of $m(u, v)$, we get

$$
m^{\nabla u}(u, v) \geq-h(u, v) \omega(q(u, v)) \omega(L(m(u, v)))
$$

which gives

$$
\frac{m^{\nabla u}(u, v)}{\omega(L(m(u, v)))} \geq-h(u, v) \omega(q(u, v))
$$


The last inequality and (44) by taking nabla integrate from $u$ to $\infty$ imply that

$$
m(u, v) \leq G^{-1}\left(G\left(\int_{u}^{\infty} h(s, v) \omega(a(s, v) q(s, v)) \nabla s\right)+\int_{u}^{\infty} h(s, v) \omega(q(s, v)) \nabla s\right) .
$$

Note that $b(u, v) \leq m(u, v)$ and a combination from (55)-(57) gives the desired bound in (43). The subdomain $u^{*}, v^{*} \in\left[u_{0}, \infty\right)_{T}$ is obvious.

Remark 2.9 As a special case of delta derivative on time scales, if we take $\alpha=1, h=0$, $g=k(s, t, \tau, \varepsilon)$, and $a(u, v)=c$, then Theorem 2.8 reduces to Theorem 4 due to Pachpatte [26].

\section{Application}

In this section, we mention an immediate application of Theorem 2.3 to study the boundedness of the following hyperbolic partial finite difference equation:

$$
[\Omega(x(u, v))]^{\nabla u}=F\left(u, v, x(u, v), \int_{u}^{\infty} \int_{v}^{\infty} M(s, t, x(s, t)) \nabla t \nabla s\right)+n(u, v)
$$

such that

$$
\Omega(x(\infty, v))=\beta(v),
$$

where $\Omega \in C^{l}\left(\mathbb{R}_{+}, \mathbb{R}_{+}\right), F:\left[u_{0}, \infty\right)_{\mathbb{T}} \times\left[u_{0}, \infty\right)_{\mathbb{T}} \times \mathbb{R} \times \mathbb{R} \rightarrow \mathbb{R}, n:\left[u_{0}, \infty\right)_{\mathbb{T}} \times\left[u_{0}, \infty\right)_{\mathbb{T}} \rightarrow \mathbb{R}$, $M:\left[u_{0}, \infty\right)_{\mathbb{T}} \times\left[u_{0}, \infty\right)_{\mathbb{T}} \times \mathbb{R} \rightarrow \mathbb{R}$, and $\beta(v): \mathbb{R}_{+} \rightarrow \mathbb{R}$.

The following example deals with the boundedness on the solutions of (58).

Example Suppose that $x(u, v)$ is a solution of (58) with (59). Also, assume that the functions are defined and continuous on their respective domains of definitions and satisfy the conditions $|F(u, v, x, y)| \leq L[g(x, y)|x+y|],|M(u, v, x)| \leq h(u, v) \omega|x|, \mid \Omega(x(\infty, v))+$ $\int_{u}^{\infty} n(s, v) \nabla s \mid \leq a(u, v)+\int_{u}^{\infty} \int_{v}^{\infty} \Omega^{\prime}(|x(s, t)|) f(s, t) \Psi(\Omega|x(s, t)|) \nabla t \nabla s$. Therefore the estimate

$$
\begin{aligned}
|x(u, v)| \leq & q(u, v) \Omega^{-1}\left[a(u, v)+L\left[F^{-1}[F(B(u, v))\right.\right. \\
& \left.\left.\left.+\int_{u}^{\infty} g(s, v)\left[q(s, v)+\int_{s}^{\infty} \int_{v}^{\infty} h(\tau, \eta) \omega(q(\tau, \eta)) \nabla \eta \nabla \tau\right] \nabla s\right]\right]\right],
\end{aligned}
$$

where $a(u, v), f(u, v), g(u, v), h(u, v), \Psi, \Omega^{\prime}, \omega, q(u, v), F^{-1}, B(u, v)$ are as mentioned in Theorem 2.3. In fact, from (58) we get

$$
\begin{aligned}
\Omega(x(u, v)) \leq & \Omega(x(\infty, v)) \\
& +\int_{u}^{\infty}\left[F\left(s, v, y(s, v), \int_{s}^{\infty} \int_{v}^{\infty} M(\tau, t, x(\tau, t)) \nabla t \nabla \tau\right)\right] \nabla s \\
& +\int_{u}^{\infty} n(s, v) \nabla s
\end{aligned}
$$


for $(u, v) \in\left[u_{0}, \infty\right)_{\mathbb{T}} \times\left[u_{0}, \infty\right)_{\mathbb{T}}$, so that

$$
\begin{aligned}
|\Omega(x(u, v))| \leq & a(u, v)+\int_{u}^{\infty} \int_{v}^{\infty} \Omega^{\prime}(|x(s, t)|) f(s, t) \Psi(\Omega(|x(s, t)|)) \nabla t \nabla s \\
& +L\left[\int_{u}^{\infty} g(s, v)\left[|x(s, v)|+\int_{s}^{\infty} \int_{v}^{\infty} h(\tau, \eta) \omega(|x(\tau, \eta)|) \nabla \eta \nabla \tau\right] \nabla s\right] .
\end{aligned}
$$

Now, a suitable application of Theorem 2.3 to the above inequality yields the required estimate (60). The uniqueness and continuous dependence of the solutions of (58) can also be discussed under some suitable conditions by using our results. To save space, we omit the details here.

\section{Acknowledgements}

I take the opportunity to thank the anonymous referees for their kind feedback and valuable guidance that helped to make the paper more authentic.

Funding

Not applicable.

Availability of data and materials

Not applicable.

\section{Competing interests}

The author declares that there are no financial and non-financial competing interests.

\section{Authors' contributions}

The author read and approved the final manuscript.

\section{Publisher's Note}

Springer Nature remains neutral with regard to jurisdictional claims in published maps and institutional affiliations.

Received: 22 March 2019 Accepted: 11 June 2019 Published online: 21 June 2019

\section{References}

1. Abdeldaim, A., El-Deeb, A.A.: On generalized of certain retarded nonlinear integral inequalities and its applications in retarded integro-differential equations. Appl. Math. Comput. 256, 375-380 (2015)

2. Abdeldaim, A., El-Deeb, A.A., Agarwal, P., El-Sennary, H.A.: On some dynamic inequalities of Steffensen type on time scales. Math. Methods Appl. Sci. 41, 4737-4753 (2018)

3. Agarwal, R., Bohner, M., Peterson, A.: Inequalities on time scales: a survey. Math. Inequal. Appl. 4(4), 535-557 (2001)

4. Atici, F.M., Guseinov, G.S.: On Green's functions and positive solutions for boundary value problems on time scales. J. Comput. Appl. Math. 141(1-2), 75-99 (2002)

5. Bohner, M.: Partial differentiation on time scales. In: Multivariable Dynamic Calculus in Time Scales, pp. 303-447 (2016)

6. Bohner, M., Peterson, A.: Advances in Dynamics Equations on Time Scales. Birkhäuser, Boston (2003)

7. El-Deeb, A.A.: Some Gronwall-Bellman type inequalities on time scales for Volterra-Fredholm dynamic integral equations. J. Egypt. Math. Soc. 26(1), 1-17 (2018)

8. El-Deeb, A.A., Cheung, W.S.: A variety of dynamic inequalities on time scales with retardation. J. Nonlinear Sci. Appl. 11(10), 1185-1206 (2018)

9. El-Deeb, A.A., El-Sennary, H.A., Cheung, W.S.: Some reverse Holder inequalities with Specht's ratio on time scales. J. Nonlinear Sci. Appl. 11(4), 444-455 (2018)

10. El-Deeb, A.A., El-Sennary, H.A., Nwaeze, E.R.: Generalized weighted Ostrowski, trapezoid and Gruss type inequalities on time scales. Fasc. Math. 60, 123-144 (2018)

11. El-Deeb, A.A., Xu, H., Abdeldaim, A.: Some dynamic inequalities on time scales. Adv. Differ. Equ. 2019, 130 (2019)

12. El-Owaidy, H., Ragab, A.A., Abuleela, W., El-Deeb, A.A.: On some new nonlinear integral inequalities of Gronwall-Bellman type. Kyungpook Math. J. 54, 555-575 (2014)

13. Ferreira, R.A.C., Torres, D.F.M.: Generalized retarded integral inequalities. Appl. Math. Lett. 22, 876-881 (2009)

14. Haidong, L.: A class of retarded Volterra-Fredholm type integral inequalities on time scales and their applications. J. Inequal. Appl. 2017, 293 (2017)

15. Hilger, S.: Analysis on measure chains-a unified approach to continuous and discrete calculus. Results Math. 18, 18-56 (1990)

16. Kim, Y.H.: Gronwall, Bellman and Pachpatte type integral inequalities with applications. Nonlinear Anal., Theory Methods Appl. 71(12), e2641-e2656 (2009)

17. Li, L., Meng, F., Ju, P.: Some new integral inequalities and their applications in studying the stability of nonlinear integro differential equations with time delay. J. Math. Anal. Appl. 377, 853-862 (2011) 
18. Li, W.N.: Some new dynamic inequalities on time scales. J. Math. Anal. Appl. 319(2), 802-814 (2006)

19. Li, W.N.: Some Pachpatte type inequalities on time scales. Comput. Math. Appl. 57, 275-282 (2009)

20. Li, W.N.: Some integral inequalities useful in the theory of certain partial dynamic equations on time scales. Comput. Math. Appl. 61, 1754-1759(2011)

21. Li, W.N.: Nonlinear integral inequalities in two independent variables on time scales. Adv. Differ. Equ. 2011, Article ID 283926 (2011)

22. Ma, Q.H., Pecaric, J.: The bounds on the solutions of certain two-dimensional delay dynamic systems on time scales. Comput. Math. Appl. 61, 2158-2163 (2011)

23. Mi, Y.: A generalized Gronwall Bellman type delay integral inequality with two independent variables on time scales. J. Math. Inequal. 11(4), 1151-1160 (2017)

24. Oguntuase, J.A.: On integral inequalities of Gronwall-Bellman-Bihari in several variables. J. Inequal. Pure Appl. Math. 12, Article ID 20 (2000)

25. Pachpatte, B.G.: A note on integral inequalities of Bellman-Bihari type. J. Math. Anal. Appl. 49, 295-301 (1975)

26. Pachpatte, D.B.: Estimates of certain integral inequalities on time scales. J. Math. 2013, Article ID 902087,5 pages (2013)

27. Tian, Y., El-Deeb, A.A., Meng, F.: Some nonlinear delay Volterra-Fredholm type dynamic integral inequalities on time scales. Discrete Dyn. Nat. Soc. 2018, Article ID 5841985 (2018)

28. Wang, J., Meng, F., Gu, J.: Estimates on some power nonlinear Volterra-Fredholm type dynamic integral inequalities on time scales. J. Inequal. Appl. 2017, Article ID 257 (2017)

\section{Submit your manuscript to a SpringerOpen ${ }^{\circ}$ journal and benefit from:}

- Convenient online submission

Rigorous peer review

Open access: articles freely available online

- High visibility within the field

- Retaining the copyright to your article

Submit your next manuscript at $\mathbf{s p r i n g e r o p e n . c o m ~}$ 